PROCEEDINGS OF THE

AMERICAN MATHEMATICAL SOCIETY

Volume 136, Number 9, September 2008, Pages 3231-3238

S 0002-9939(08)09403-3

Article electronically published on May 5, 2008

\title{
THE REMAINDER IN ASYMPTOTIC INTEGRATION
}

\author{
HORST BEHNCKE \\ (Communicated by Carmen C. Chicone)
}

\begin{abstract}
Quantitative estimates for the remainder terms in Levinson's Theorem are provided. This gives a precise meaning to the idea that small perturbations should result in small remainder terms.
\end{abstract}

\section{INTRODUCTION}

One of the most useful results in asymptotic integration theory is Levinson's Theorem. It states that the solutions of a linear differential system

$$
y^{\prime}=(\wedge(x)+R(x)) y, \quad \wedge=\operatorname{diag}\left(\lambda_{1}(x), \ldots, \lambda_{n}(x)\right)
$$

on $[a, \infty)$ behave almost like the solutions of the unperturbed diagonal system $y^{\prime}=\wedge y$, if the $\lambda_{i}$ satisfy a dichotomy condition and if the perturbing matrix $R$ is small. Then the solutions may be written as

$$
y_{k}=\left(e_{k}+r_{k}(x)\right) \exp \mu_{k}(x) \quad \text { with } \mu_{k}(x)=\int_{a}^{x} \lambda_{k}(t) \mathrm{dt} .
$$

Here $e_{k}, k=1, \ldots, n$, stands for the $k$-th unit vector and by Levinson's Theorem one has $r_{k}=o(1)$. Meanwhile this theorem has been extended in many ways. A nearly complete survey is given in the book of Eastham [2] which is almost solely devoted to this theorem and its applications. While the above form of Levinson's Theorem suffices in most cases, a better control of the remainder term $r_{k}(x)$ is advisable. Levinson's result also shows that the diagonal influences the solutions mainly via the exponential factors, while the off-diagonal terms are responsible for the remainder. Thus an integrable perturbation of the diagonal can easily be transformed away by the exponential of this diagonal, which then only causes relatively bounded changes in the off-diagonal terms. This will be used repeatedly without further comments. It is plausible that small perturbation terms $R$ imply the smallness of the remainders $r_{k}$ likewise. The aim of this paper is to make this more precise. The starting point for this is a special version of a generalization of the Hartman-Wintner-Theorem, which has been shown by Remling and this author [1.

Theorem 1. Assume for $i \neq j, i, j=1, \ldots, n$,

(a) $\left|\operatorname{Re}\left(\lambda_{i}(x)-\lambda_{j}(x)\right)\right| \geq c x^{-\alpha_{i j}}, \quad c>0, \alpha_{i j}=\alpha_{j i}<1$,

(b) $R_{i j} x^{\beta_{i j}} \in \mathcal{L}^{p}, R_{i i} \in \mathcal{L}^{1}, p \geq 1$,

(c) $p^{\prime} \beta_{i j} \geq \alpha_{i}$, where $1 / p+1 / p^{\prime}=1$ and $\alpha_{i}=\max _{j \neq i} \alpha_{i j}$.

Received by the editors July 30, 2007.

2000 Mathematics Subject Classification. Primary 34E10.

(C)2008 American Mathematical Society Reverts to public domain 28 years from publication 
Then (11) has solutions of the form (2), with $\mu_{i}(x)=\int_{a}^{x} \tilde{\lambda}_{i}(t) d t$ and $\left(\lambda_{i}(x)-\tilde{\lambda}_{i}(x)\right)=$ $o\left(x^{-\alpha_{i}}\right)$.

The fact that one has to use different exponents $\tilde{\lambda}$ in this result is a consequence of the indirect effect of "large" coefficients of $R$ on other matrix elements. This, in fact, is the main obstacle to a proper estimate of the remainder terms and the asymptotics. The condition $R_{i i} \in \mathcal{L}^{1}$ can be achieved by absorbing the largest part of $R_{i i}$ into $\lambda_{i}$. That this has a negligible effect follows from [1, Lemma 4.3].

Here we will show that with the assumptions of the theorem above and $p^{\prime} \beta_{i j}>\alpha_{i}$ one almost has

$$
r_{k, i} \in \mathcal{L}^{p} x^{-\left(\beta_{i k}-\alpha_{i} / p^{\prime}\right)}, \quad i \neq k,
$$

where $r_{k, i}$ stands for the $i$-th component of the $k$-th remainder term. Of course, this naive, though plausible, statement will have to be made more precise. This result is based on a power scale. Thus, in a second part we will also indicate a result based on more general scales.

This paper consists of two sections: the introduction and the main part II, the $(1+Q)$-transformation. Extensions of the result will be indicated at the end.

\section{The $(1+Q)$-TRANSFORMATION}

In this section we will first analyze the repeated $(1+Q)$-transformations. In a second step we study the solutions of the reduced system. The proof of the Hartman-Wintner Theorem and this result is based on the $(1+Q)$-transformation, $y=(1+Q) z$, which leads to

$$
z^{\prime}=\left[\wedge+(1+Q)^{-1}\left(\wedge Q-Q \wedge+R-Q^{\prime}+R Q\right)\right] z .
$$

Actually we will use what we will we call the off diagonal $(1+Q)$-transformation. With this approach the diagonal part $d g R$ of $R$ is adjoined to $\wedge$ and (3) is used for $\tilde{\wedge}=\wedge+d g R$ and $\tilde{R}=R-d g R$. Thus we get

$$
z^{\prime}=\left[\tilde{\wedge}+(1+Q)^{-1}\left(\tilde{\wedge} Q-Q \tilde{\wedge}+\tilde{R}-Q^{\prime}+\tilde{R} Q\right] z,\right.
$$

and we have to determine $Q$ with $d g Q=0$ so that $Q(x) \rightarrow 0$ for $x \rightarrow \infty$ and

$$
Q^{\prime}=\tilde{\wedge} Q-Q \tilde{\wedge}+\tilde{R} .
$$

Since $d g \tilde{R}=0$, (4) reduces to a system of $n(n-1)$ ordinary linear differential equations of first order,

$$
q_{i j}^{\prime}=\left(\lambda_{i}-\lambda_{j}\right) q_{i j}+r_{i j}, \quad i, j=1, \ldots, n, i \neq j,
$$

which we write for short as

$$
q^{\prime}=\lambda q+r .
$$

The solutions, which are small at infinity, are given by

$$
\begin{array}{ll}
q(x)=-\int_{x}^{\infty} \exp (\mu(x)-\mu(t)) r \mathrm{dt} & \text { if } \operatorname{Re} \lambda \geq 0, \mu=\int_{a}^{x} \lambda(t) \mathrm{dt}, \\
q(x)=\int_{a}^{x} \exp (\mu(x)-\mu(t)) r \mathrm{dt} \quad & \text { if } \operatorname{Re} \lambda \leq 0 .
\end{array}
$$


Here we have deleted the indices and written $\lambda$ for $\lambda_{i}-\lambda_{j}$ and $\mu$ for $\mu_{i}-\mu_{j}$. The key tool in [1] was the estimates of integral operators of the form $T_{i}: \mathcal{L}^{p}([a, \infty]) \rightarrow$ $\mathcal{L}^{\infty}([a, \infty])$ with

$$
\begin{aligned}
& T_{1} f(x)=\int_{x}^{\infty} h_{2}^{-1}(x) \exp (\nu(x)-\nu(t)) h_{1}(t) f(t) d t \\
& T_{2} f(x)=\int_{a}^{x} h_{2}^{-1}(x) \exp (-\nu(x)+\nu(t)) h_{1}(t) f(t) d t
\end{aligned}
$$

which arise in connection with (7).

Lemma 1. Assume $p>1$ and let $p^{\prime}$ be the conjugate index of $p$. Assume further that $\nu$ and $h_{1}, h_{2}$ are positive and twice differentiable with $0<\nu^{\prime}(x)=\gamma(x)$. Then the operators $T_{1}$ and $T_{2}$ are bounded operators from $\mathcal{L}^{p}$ to $\mathcal{L}_{0}^{r}, r \geq p$ if $h_{2} \geq$ $h_{1} \gamma^{-\sigma_{i}}, i=1,2$, with $\frac{\sigma_{1}}{p^{\prime}}+\frac{\sigma_{2}}{r}=1$ and if $\left(\ln h_{i}\right)^{\prime} \gamma^{-1}=o(1)$. The result is also valid for $r=\infty$ if $\sigma_{i}=p^{\prime}$ for $i=1,2$. Here $\mathcal{L}_{0}^{p}$ denotes the set of all $f$ in $\mathcal{L}^{p}$, which vanish at infinity.

Proof. The Schnur test of Okikiolu [3, 4.1], $i=1,2$ with $\phi=1$ and a transformation of $\nu \rightarrow x$, reduces the problem to the boundedness of

$$
\begin{aligned}
& I_{1}=\int_{x}^{\infty} h_{2}(x)^{\sigma} e^{x \sigma-t \sigma} h_{2}(t)^{-\sigma} d t \quad \text { and } \\
& I_{2}=\int_{a}^{x} h_{2}(x)^{\sigma} e^{-x \sigma+t \sigma} h_{2}(t)^{\sigma} d t .
\end{aligned}
$$

Now write $\ln h_{2}=k$ and introduce the new variable $s(t)=t+k(t)$. This absorbs $h_{2}$ into the exponential, and the boundedness of $I_{1}$ and $I_{2}$ follows trivially.

For us only the cases $r=p, \sigma_{1}=\sigma_{2}=1$ and $r=\infty, \sigma_{1}=\sigma_{2}=p^{\prime}$ are important. The $p=\infty$ result follows simply from an $\mathcal{L}^{1}$-estimate of the kernel. In the first step with power scales we will use the lemma with $\nu(x)=D x^{1-\alpha}$ and $h_{i}(x)=x^{\beta}$. Since we want the decay properties to improve with each $(1+Q)$-transform, we strengthen the condition of Theorem 1 slightly to

$$
p^{\prime} \beta_{i j}>\alpha_{i}
$$

and set $\delta=\min \left(\beta_{i j}-\alpha_{i} / p^{\prime}\right)$.

The first $(1+Q)$-transformation. After the $(1+Q)$-transformation the $z$-equation has the form $z^{\prime}=\left(\tilde{\wedge}+R_{1}\right) z$ with $R_{1}=(1+Q)^{-1} R Q=\sum(-1)^{k} Q^{k} R Q$. It follows from Lemma 1 that $q_{i j} x^{\left(\beta_{i j}-\alpha_{i j / p^{\prime}}\right)} \in \mathcal{L}_{0}^{\infty}$; i.e. it is bounded and vanishes at infinity if $r_{i j} x^{\beta_{i j}} \in \mathcal{L}^{p}$. Since the terms $\frac{\alpha_{i j}}{p^{\prime}}$ keep appearing repeatedly, we will abridge them by $\alpha_{i j}^{\prime}$. A typical matrix element of $Q^{l} R Q$ is made up of terms $q_{i k_{1}} q_{k_{1} k_{2}} \ldots r_{k_{l} k_{l+1}} q_{k_{l+1}, j}$ with $i \neq k_{1} \neq k_{2} \neq \ldots$. Such an expression will be an element of $\mathcal{L}^{p} x^{-\gamma}$ with

$$
\begin{aligned}
\gamma & =\beta_{i k_{1}}-\alpha_{i k_{1}}^{\prime}+\beta_{k_{1}, k_{2}}-\ldots-\alpha_{k_{l-1}, k_{l}}^{\prime}+\beta_{k_{l}, k_{l+1}}+\beta_{k_{l+1}, j}-\alpha_{k_{l+1}, j} \\
& \geq \beta_{i k_{1}}+(l+1) \delta .
\end{aligned}
$$

Thus each $Q$-factor improves $R$ on the average by $x^{-\delta}$ and $p^{\prime} \beta_{i j}>\alpha_{i}$ is still valid. The above sum could be interpreted as a charge and path length in a weighted graph with vertices $1, \ldots, n$. Thus the appropriate $\gamma$ for $\left(Q^{l} R Q\right)_{i j}$ would be the minimum of such sums, which we denote by $\gamma(l+1, i, j) \geq \beta_{i}+(l+1) \delta$ where 
$\beta_{i}=\min _{k} \beta_{i k}$. Now repeat this transformation. For this, $\left((1+Q)^{-1} R Q\right)_{i i}$ will be adjoined to $\tilde{\lambda}_{i}$. Thus the new diagonal element is

$$
\lambda_{i}^{(1)}=\lambda_{i}+R_{i i}+\left((1+Q)^{-1} R Q\right)_{i i} .
$$

As before we see that $\left(Q^{l} R Q\right)_{i i}$ is made up of terms $q_{i k_{1}} q_{k_{1}, k_{2}} \ldots r_{k_{l}, k_{l+1}} q_{k_{l+1}, i} \in$ $\mathcal{L}^{p} x^{-\gamma}$, where $\gamma$ can be evaluated as above. It follows again from 1, Lemma 4.2, 4.3] that (9) is still valid because for the diagonal terms we may work modulo integrable expressions [1]. In general one cannot expect $\left(Q^{l} R Q\right)_{i i}=o\left(\lambda_{i}\right)$, as examples with $\lambda_{i}=0$ show. Thus $\lambda_{i i}^{(1)}-\lambda_{i}=o\left(x^{-\alpha_{i}-\delta}\right)$ is the best to expect. Nonetheless, the transformed system still satisfies the conditions of the theorem with possibly new exponents. With our stronger condition (8), however, we even have $\left|\lambda_{i}^{(1)}-\lambda_{i}\right| \leq C x^{-\alpha-\eta}$ for some $\eta>0$.

Repeated $(1+Q)$-transformation. Now repeat these transformations. Altogether one obtains

$$
y=\left(1+Q_{1}\right)\left(1+Q_{2}\right) \ldots\left(1+Q_{l}\right) v_{l}, \quad v_{l}^{\prime}=\left(\wedge_{l}+R_{l}\right) v_{l} .
$$

Here $Q_{1}=Q, R_{1}=\left(1+Q_{1}\right)^{-1} R Q_{1}$. $Q_{2}$ is based on $R_{1}$ via (6) or (7). Thus $R_{2}=\left(1+Q_{2}\right)^{-1} R_{1} Q_{2}, \ldots$ This shows with (6), (7) and the lemma that matrix elements associated to $R_{2}$ can be estimated as $r_{i j}^{(2)} \in \mathcal{L}^{p} x^{-\beta_{i}-3 \delta}$.

Along the same lines we see that elements of $R_{3}$ satisfy $r_{i j}^{(3)} \in \mathcal{L}^{p} x^{-\beta_{i}-7 \delta}$. More generally this shows that elements of $Q_{l}$ and $R_{l}$ have associated exponents of at least $-2^{(l-1)} \delta$ and $-\beta_{i}-\left(2^{l}-1\right) \delta$. We note that $f \in \mathcal{L}^{p} \cdot x^{-\beta}$ is integrable if $p^{\prime} \beta>1$. We also note that the repeated $(1+Q)$-transforms tend to even out the exponents. Thus we finally arrive at a system of the form

$$
v^{\prime}=(\wedge+R) v \quad \text { with } R_{i i}=0, \quad R_{i j} \in \mathcal{L}^{p} x^{-\beta},
$$

with $\beta>\beta_{i j}+1 / p^{\prime}$. For simplicity we have adjusted and simplified the notation.

Estimates for the solution. We will now show in a second step that

$$
v_{k}=\left(e_{k}+r_{k}(x)\right) \exp \mu_{k} \quad \text { with } r_{k, i} \in \mathcal{L}^{p} x^{-\beta}, k \neq i \quad \text { and } \quad r_{k k}(x) \rightarrow 0 .
$$

For the proof of (12) we follow the proof and notation of Levinson's Theorem in [2, 1.4.1]. Then the fundamental matrix $\phi$ of the diagonal system is decomposed according to the asymptotics of the eigenfunctions $\phi=\phi_{1}+\phi_{2}$ so that $\left|\phi_{1}(x) \phi^{-1}(t)\right| \leq C_{1}$ for $a \leq t \leq x$ and $\left|\phi_{2}(x) \phi^{-1}(t)\right| \leq C_{2}$ for $a \leq x \leq t$. Then the $k$-th solution satisfies the integral equation

$$
Z(x)=e_{k}+\phi_{1}(x) \int_{a}^{x} \phi^{-1}(t) R Z(t) \mathrm{dt}-\int_{x}^{\infty} \phi_{2}(x) \phi^{-1}(t) R(t) Z(t) \mathrm{dt} .
$$

With $Z^{1}=e_{k}$ this equation can be solved iteratively [2] by

$$
\begin{aligned}
Z^{(m+1)}(x)=e_{k}+\phi_{1}(x) \int_{a}^{x} \phi^{-1}(t) R Z^{(m)} & (t) \mathrm{dt} \\
& -\int_{x}^{\infty} \phi_{2}(x) \phi^{-1}(t) R(t) Z^{(m)}(t) \mathrm{dt} .
\end{aligned}
$$


Reverting to our notation this means for $i \neq k$

$$
\begin{aligned}
& r_{k, i}^{(m+1)}(x)= \int_{a}^{x} \exp \left(\nu_{i}(x)-\nu_{i}(t)\right) \sum R_{i j}(t) r_{k j}^{(m)}(t) \mathrm{dt} \\
& \quad \text { if } \operatorname{Re} \nu_{i}(x) \rightarrow-\infty, \\
& r_{k, i}^{(m+1)}(x)=-\int_{x}^{\infty} \exp \left(\nu_{i}(x)-\nu_{i}(t)\right) \sum R_{i j}(t) r_{k j}^{(m)}(t) \mathrm{dt}, \quad i \neq k, \\
& \quad \text { if } \operatorname{Re} \nu_{i}(x) \rightarrow \infty .
\end{aligned}
$$

Here one should remember that $\nu_{i}$ actually arises from $\lambda_{i}-\lambda_{k}$. The second expression in (15) can also be used for $i=k$. It is not difficult to show that $Z^{(m)}$ is bounded [2]. Moreover $Z_{i}^{(m)}=r_{k i}^{(m)}, i \neq k$. The integral transforms encountered in (15) can easily be brought into the form $T_{1}$ or $T_{2}$ of the lemma. Thus we see by induction with the aid of Lemma 1 that $r_{k, i}^{(m)} \in \mathcal{L}^{p} x^{-\beta}, k \neq i$. This also shows that the leading term in (15) arises from $R_{i k} Z_{k}^{(m)}$ because all other components decay faster. Putting all things together we get

$$
y=\left(1+Q_{1}\right) \ldots\left(1+Q_{l}\right)\left(\left(1+r_{k k}(x)\right) e_{k}+\sum_{i \neq k} r_{k, i} e_{i}\right) \exp \mu_{k} .
$$

Modulo $\mathcal{L}^{p} x^{-\beta}$ this amounts to

$$
y \equiv\left(1+Q_{1}\right) \ldots\left(1+Q_{l}\right) e_{k}\left(1+r_{k k}\right) \exp \mu_{k},
$$

so that it remains to compute the matrix product. For this we begin with $\left(Q_{i_{1}} \ldots\right.$ $\left.Q_{i_{l}}\right)_{i k}$. Each of these terms can be estimated by $\mathcal{L}^{p} x^{-\gamma}$, where $\gamma$ can be evaluated as above. This will also hold for the product of such terms. Thus $\left(\left(1+Q_{1}\right) \ldots(1+\right.$ $\left.\left.Q_{l}\right)-1\right)_{i k}$ will likewise have this form.

The repeated $(1+Q)$-transformation will reduce the remainder terms $R_{1}, R_{2}, \ldots$ on the average. Thus $Q_{1}, Q_{2}, \ldots$ will decrease likewise and on the average only the first few factors in (16) will determine the growth of the remainder terms. Similarly we can expect that only the first few transformations produce nonintegrable, i.e. significant contribution to the diagonal. To make this more precise assume

$$
\begin{aligned}
\beta_{i k}- & \alpha_{i / p^{\prime}}<\left(\beta_{i k_{1}}-\alpha_{i k_{1}}^{\prime}+\left(\beta_{k_{1}, k_{2}}-\alpha_{k_{1}, k_{2}}^{\prime}\right)+\ldots\right. \\
& +\left(\beta_{k_{l}, k}-\alpha_{k_{l-1} k_{l}}^{\prime}\right)+\beta_{k_{l}, k} \quad \text { for } l=2,3, \ldots, k_{1} \neq k_{2} \neq \ldots \neq k_{l} \neq k .
\end{aligned}
$$

Moreover assume for the diagonal terms

$$
\begin{array}{r}
\left(\beta_{k k_{1}}-\alpha_{k k_{1}}^{\prime}\right)+\left(\beta_{k_{1} k_{2}}-\alpha_{k_{1} k_{2}}^{\prime}\right)+\ldots+\left(\beta_{k_{l-1}, k_{l}}-\alpha_{k_{l-1} k_{1}}^{\prime}\right)+\beta_{k_{l} k}>1 / p^{\prime} \\
\text { for all } k_{1} \neq k_{2} \neq \ldots \neq k \text { and } l \geq 3 .
\end{array}
$$

Theorem 2. Consider the system (1) on $[a, \infty)$ with

a) $\left|\operatorname{Re}\left(\lambda_{i}(x)-\lambda_{j}(x)\right)\right| \geq c x^{-\alpha_{i j}}$ with $c>0,0 \leq \alpha_{i j}=\alpha_{j i}<1$,

b) $R_{i j} x^{\beta_{i j}} \in \mathcal{L}^{p}, \quad i \neq j$,

c) $p^{\prime} \beta_{i j}>\alpha_{i}=\max \alpha_{i j}$.

Moreover assume (17) and (18). Then the $k$-th solution of (11) has the form (2) with $r_{k, i} \in \mathcal{L}^{p} \cdot x^{-\left(\beta_{i k}-\alpha_{i} / p^{\prime}\right)}$ and $\lambda$ replaced by $\tilde{\lambda}_{k}=\lambda_{k}+\left(R_{1}\right)_{k, k}$ with $R_{1}=R Q-Q R Q$.

Remark. If (17) and (18) do not hold, similar results can easily be derived along the same lines. Note that this result separates the perturbing effects of "large" coefficients of $R$ on the diagonal (18) and the off diagonal (17). In the first part 
of the proof $l,(1+\mathrm{Q})$-transformations were used in order to reach (11). Thus $l$ will in general be proportional to $\delta^{-1}$. On the other hand the precise number $l$ is irrelevant for the proof since only (16) is relevant for the remainder.

Proof. It follows from (17) that only $Q_{1}$ contributes essentially, modulo $\mathcal{L}^{p}$. $x^{-\beta_{i k}-\alpha_{i} / p^{\prime}}$, to $r_{k, i}$. As noted before, the essential contribution to the diagonal comes from the nonintegrable part of $\left(R_{l}\right)_{k, k}$. These can arise from $R_{k, k}$ and $\left(R_{1}\right)_{k, k}$ only while $R_{2}, R_{3}, \ldots$ contribute integrable perturbations of the diagonal.

This result admits a number of generalizations. Thus it is clear that it holds for individual $y_{k}$. Secondly one could allow the $p$ 's to vary, and finally (17) and (18) could be weakened at the cost of more contributions to the diagonal and offdiagonal. A closer look at the proof also shows the the required inequalities need not be strict in all cases, because what counts are the matrix elements $Q_{i k_{1}} Q_{k_{1} k_{2}} \ldots Q_{k_{l} j}$ with $k_{1} \neq k_{2} \neq \ldots \neq k_{l}$.

The $(1+Q)$-transformation has the advantages that it is easy to use and requires very few additional conditions. Its disadvantage is that it requires fairly strong conditions on the real part. On the other hand, small $\operatorname{Re}\left(\lambda_{i}-\lambda_{j}\right)$ but larger $\left|\lambda_{i}-\lambda_{j}\right|$ could be handled by repeated diagonalizations. This, however, requires additional smoothness of the coefficients. The simple example

$$
u^{\prime}=\left(\begin{array}{cc}
i & r \\
0 & -i
\end{array}\right) u,
$$

however, quickly shows the limitations of such possible extensions. Thus the $(1+Q)$ transformation method will also work in the large $\operatorname{Im}\left(\lambda_{i}-\lambda_{j}\right)$ case if nonresonance conditions between $R$ and the diagonal $\wedge$ hold. In lowest order this corresponds to the absence of Wigner-van-Neumann type terms.

Lemma 1 has already been stated in a form which allows different scales $\nu_{i}^{\prime}$ and $h_{i}$. However, since a formulation of the corresponding generalisation of Theorem 2 would be rather technical, we abstain from such a result. After all, it is the method of proof which can be used in concrete cases. It is advantageous, however, to use an exponential presentation in Lemma 1 and its applications; i.e. one should write $h_{i}=\exp k_{i}$ and $v^{\prime}=\exp l$ so that $k_{2}=k_{1}-l$. Then the conditions of Lemma 1 are $k^{\prime}, l^{\prime}=o(\gamma)$. The conditions of the theorem will then be $p^{\prime} k_{i j} \geq(1+\epsilon) v_{i}$.

The following example illustrates an application of this method, but it also shows the limitations if, as in this case, $\operatorname{Re} \lambda_{3}=\operatorname{Re} \lambda_{4}=0$.

Consider a fourth-order operator

$$
L_{y}=y^{I V}-\left(p y^{\prime}\right)^{\prime}+q y \quad \text { on } \mathcal{L}^{2}([0, \infty)) .
$$

We will assume the coefficients to be of approximate power type; i.e. $p=p_{0} x^{\alpha}(1+$ $h), q=q_{0} x^{\beta}\left(1+k_{1}\right)+k_{2}$ with $p_{0}>0, q_{0}$ constant and $h, k_{1}, k_{2} \in \mathcal{F}_{2}$, where $\mathcal{F}_{k}=$ $\left\{f \mid f^{(l)}=o\left(x^{-l}\right), 0 \leq l \leq k\right\}$.

The standard procedure to determine the asymptotics of the eigenfunctions of $L, L y=z y$ is to transform (19) into a system [2]

(20) $u^{\prime}=\left(\begin{array}{cccc}0 & 1 & 0 & 0 \\ 0 & 0 & 0 & 1 \\ q_{0} & 0 & 0 & 0 \\ 0 & p & -1 & 0\end{array}\right) u=\mathcal{C} u \quad$ with $u=\left(y, y^{\prime}, p y^{\prime}-y^{\prime \prime \prime}, y^{\prime \prime}\right)^{t}, q_{0}=q-z$, 
and to diagonalize this system. For this one needs the eigenvalues of $\mathcal{C}$. They are the roots of $\lambda^{4}-p \lambda^{2}+q_{0}=0$. A nontrivial but not too complicated case arises if $p$ is dominant and positive:

$$
p_{0}>0,2 \alpha>\beta \geq 0, \quad 2+\beta>\alpha .
$$

In this case $\mathcal{C}$ has eigenvalues of two different sizes, $\lambda_{1}=-\lambda_{2} \sim p^{\frac{1}{2}}$ and $\lambda_{3}=-\lambda_{4} \sim\left(\frac{q_{0}}{p}\right)^{\frac{1}{2}}$. If $\lambda$ is an eigenvalue of $\mathcal{C}$, the corresponding eigenvector is $\left(1, \lambda, p \lambda-\lambda^{3}, \lambda^{2}\right)^{t}=\varrho(\lambda)$. Thus the matrix $T$ formed with the eigenvectors $M_{i}^{-\frac{1}{2}} \varrho\left(\lambda_{i}\right), M_{i}=4 \lambda_{i}^{2}-2 p \lambda_{i}$ columns diagonalizes $\mathcal{C}$. Thus the transformation $T v=u$ gives

$$
v^{\prime}=\left(T^{-1} \mathcal{C} T-T^{-1} T^{\prime}\right) v=\left(\wedge-T^{-1} T\right) v, \quad \wedge=\operatorname{diag} \cdot \lambda_{i} .
$$

The matrix elements of $T^{-1} T^{\prime}$ are given by

$$
\left(T^{-1} T^{\prime}\right)_{i j}=\left(\lambda_{j}-\lambda_{i}\right)^{-1} M_{i}^{-\frac{1}{2}} M_{j}^{-\frac{1}{2}}\left(p^{\prime} \lambda_{i} \lambda_{j}-q^{\prime}\right), i \neq j,\left(T^{-1} T^{\prime}\right)_{i, i}=0
$$

The last relation is a consequence of the $M^{-\frac{1}{2}}$ factor with the eigenvectors. Since the system (20) is not diagonal yet, a second diagonalization with a matrix $(1+B)$ and $B_{i j}=-\left(T^{-1} T^{\prime}\right)_{i j}\left(\lambda_{j}-\lambda_{i}\right)^{-1}, i \neq j, B_{i i}=0$, can be performed, $v=(1+B) v_{1}$. To the resulting system

$$
v_{1}^{\prime}=(\tilde{\wedge}+R) v_{1}
$$

we can apply our theorem. Because of (21), $2+\beta>\alpha$, all corrections to $\wedge$ are integrable, so that we may take $\wedge=\tilde{\wedge}$. The correction terms $R$ arise from $B^{2} \wedge, B \wedge$ $B, B \cdot\left(T^{-1} T^{\prime}\right), \ldots$

The following list gives the negative $x$-exponents of the various matrix elements $A_{i j}$, and we use the abbreviation $l=1,2, s=3,4$ :

\begin{tabular}{l|lll} 
& 1,2 & $l, s$ & 3,4 \\
\hline$\left(T^{-1} T^{\prime}\right)_{i j}$ & 1 & $1+\frac{\alpha}{2}-\frac{\beta}{4}$ & 1 \\
\hline$B$ & $1+\frac{\alpha}{2}$ & $1-\frac{\beta}{4}+\alpha$ & $1+\frac{\beta-\alpha}{2}$ \\
$B^{2}$ & $2+2 \alpha-\frac{\beta}{2}$ & $2+\frac{\alpha}{2}+\frac{\beta}{4}$ & $2-\frac{\beta}{2}+2 \alpha$ \\
$R$ & $2+\frac{\alpha}{2}-\frac{\beta}{2}$ & $2+\frac{\beta}{2}-\frac{\alpha}{2}$ & $2+\frac{\beta}{2}-\frac{\alpha}{2}$ \\
$Q$ & $2+\alpha-\frac{\beta}{2}$ & $2+\frac{\beta}{2}$ & $*$
\end{tabular}

The last line lists the negative exponents of the corresponding matrix $Q=Q_{1}$. As regards $Q_{3,4}$ and $Q_{4,3}$ the exponents are $1+\frac{\beta}{2}-\frac{\alpha}{2}$ if $q-\operatorname{Re} z$ is negative or $\lambda_{3}=-\lambda_{4}$ purely imaginary. If $q_{0}-\operatorname{Re} z$ is positive, the exponent is 2 . Thus if $q_{0}>0$ and $\beta>0$, the spectrum of the associated selfadjoint operators $H$ associated to $L$ are discrete and the eigenfunction $\bmod x^{-2}$ remainder terms are given by

$$
T(1+B) e_{k} \exp \int_{a}^{x} \lambda_{k}(t) d t
$$

An interesting case arises for $q_{0}<0, \beta>0$. In this case the absolutely continuous spectrum of $H$ is $\mathbb{R}$ of multiplicity 1 . The corresponding eigenfunctions are associated to $\lambda_{3}$ and $\lambda_{4}$. Modulo $x^{-2}$ remainder terms they arise from the system restricted to the $(3,4)$ corner. Basically these functions are plane waves. Since 
$\left(Q_{1}\right)_{3,4} \sim x^{-(2+\beta-\alpha)}$ the expansion (10) above will have to include many $Q_{k}$ if $2+\beta-\alpha$ is rather small. Thus in this case $l$ would be rather large, $l \approx c \cdot(z+\beta-\alpha)^{-1}$.

For $q_{0}=0$ the absolutely continuous spectrum of $H$ is $[0, \infty)$ of multiplicity 1. Again, the corresponding eigenfunctions are associated to $\lambda_{3}$ and $\lambda_{4}$, and these eigenfunctions arise essentially (i.e. $\left.\bmod x^{-2}\right)$ from the $(3,4)$ subsystem only. The spectrum in $(-\infty, 0)$ is discrete, and the corresponding eigenfunctions $\bmod x^{-2}$ arise essentially from the $(3,4)$ subsystem.

\section{REFERENCES}

[1] H. Behncke, C. Remling: Asymptotic integration of linear differential equations. J. of Math. Analysis and Appl. 210 (1997) 585-597. MR1453193 (98i:34012)

[2] M.S.P. Eastham: The asymptotic solution of linear differential systems, in London Mathematical Society Monographs New Series, Vol. 4, Oxford Univ. Press, Oxford, 1989. MR 1006434 (91d:34001)

[3] G. Okikiolu: "Aspects of the Theory of Bounded Integral Operators in $L_{p}$ Spaces", Academic Press, New York, 1971. MR0445237 (56:3581)

Fachbereich Mathematik/Informatik, Universität Osnabrück, Osnabrück D-49069, Germany 